\title{
Analytical expressions for curved surface area of revolution and arc-length of an ellipse: A hypergeometric mechanism
}

\author{
M. I. Qureshi · Naved Akhtar ${ }^{\star}$ - Dilshad Ahamad
}

Received: 06.12.2018 / Revised: 15.09.2019 / Accepted: 19.01.2020

\begin{abstract}
In this paper analytical expressions of curved surface area of revolution of an arc between two arbitrary points lying on an ellipse about major and minor axes and the arc-length between two arbitrary points lying on an ellipse are obtained in terms of Gauss' hypergeometric function, Appell's function of first kind and Kampé de Fériet's double hypergeometric function. Moreover, we have also discussed the perimeter of orbit of some known planets and other special cases.
\end{abstract}

Keywords. Arc-length, Curved surface area, Ellipse, Generalized Hypergeometric Function, Kampé de Fériet's double Hypergeometric Function.

Mathematics Subject Classification (2010): 33C05, 33C20, 33C70, 33C75.

\section{Introduction and Preliminaries}

For the sake of conciseness of the paper we have used the following notations

$\mathbb{N}:=\{1,2,3, \ldots\} ; \mathbb{N}_{0}:=\mathbb{N} \cup\{0\}=\{0,1,2,3, \ldots\} ; \mathbb{Z}:=\{0, \pm 1, \pm 2, \pm 3, \ldots\} ;$ $\mathbb{Z}^{-}:=\{-1,-2,-3, \ldots\} ; \mathbb{Z}_{0}^{-}:=\mathbb{Z}^{-} \cup\{0\}=\{0,-1,-2,-3, \ldots\} ;$ and $\mathbb{Z}:=\mathbb{Z}_{0}^{-} \cup \mathbb{N}$, where the symbols $\mathbb{N}$ and $\mathbb{Z}$ are the set of natural numbers and set of integers respectively, the symbols $\mathbb{R}$ and $\mathbb{C}$ are the set of real numbers and set of complex numbers respectively.

\footnotetext{
* Corresponding author
}

Naved Akhtar

Department of Applied Sciences and Humanities, Faculty of Engineering and Technology, Jamia Millia Islamia (A Central University), New Delhi-110 025, India,

E-mail: naved.a86@gmail.com

M. I. Qureshi

Department of Applied Sciences and Humanities, Faculty of Engineering and Technology,

Jamia Millia Islamia (A Central University), New Delhi-110 025, India,

E-mail: miqureshi_delhi@yahoo.co.in

Dilshad Ahamad

Department of Applied Sciences and Humanities, Faculty of Engineering and Technology, Jamia Millia Islamia (A Central University), New Delhi-110 025, India,

E-mail: dlshdhmd4@gmail.com 
The Pochhammer symbol $(\alpha)_{p},(\alpha, p \in \mathbb{C})$, is defined by

$$
(\alpha)_{p}:=\frac{\Gamma(\alpha+p)}{\Gamma(\alpha)}= \begin{cases}1, & (p=0 ; \alpha \in \mathbb{C} \backslash\{0\}) \\ \alpha(\alpha+1) \cdots(\alpha+n-1), & (p=n \in \mathbb{N} ; \alpha \in \mathbb{C}) \\ \frac{(-1)^{n} k !}{(k-n) !}, & \left(\alpha=-k ; p=n ; n, k \in \mathbb{N}_{0} ; 0 \leq n \leq k\right) \\ 0, & \left(\alpha=-k ; p=n ; n, k \in \mathbb{N}_{0} ; n>k\right) \\ \frac{(-1)^{n}}{(1-\alpha)_{n}}, & (p=-n ; n \in \mathbb{N} ; \alpha \in \mathbb{C} \backslash \mathbb{Z}) .\end{cases}
$$

It being understood conventionally that $(0)_{0}=1$, and assumed tacitly that the Gamma quotient exists.

If $a, p \in \mathbb{C}$ and $r=0,1,2,3, \ldots$, then

$$
\begin{aligned}
a+p r & =\frac{a\left(\frac{a+p}{p}\right)_{r}}{\left(\frac{a}{p}\right)_{r}}, \text { such that each Pochhammer symbol is well defined. } \\
(\alpha)_{m+n} & =(\alpha)_{m}(\alpha+m)_{n}=(\alpha)_{n}(\alpha+n)_{m}, \\
\Gamma(z+1) & =z \Gamma(z) .
\end{aligned}
$$

The generalized hypergeometric function of one variable ${ }_{p} F_{q}[2, \mathbf{p . 8}]$ is defined by

$$
\begin{aligned}
{ }_{p} F_{q}\left[\begin{array}{c}
\alpha_{1}, \alpha_{2}, \ldots, \alpha_{p} ; \\
\beta_{1}, \beta_{2}, \ldots, \beta_{q} ;
\end{array}\right] & ={ }_{p} F_{q}\left[\begin{array}{c}
\left(\alpha_{p}\right) ; \\
\left(\beta_{q}\right) ;
\end{array}\right]=\sum_{n=0}^{\infty} \frac{\prod_{j=1}^{p}\left(\alpha_{j}\right)_{n}}{\prod_{j=1}^{q}\left(\beta_{j}\right)_{n}} \frac{z^{n}}{n !} \\
& =1+\sum_{n=1}^{\infty} \frac{\prod_{j=1}^{p}\left(\alpha_{j}\right)_{n}}{\prod_{j=1}^{q}\left(\beta_{j}\right)_{n}} \frac{z^{n}}{n !},
\end{aligned}
$$

where, $\left(\alpha_{p}\right)$ is a set of parameters $\alpha_{1}, \alpha_{2}, \cdots, \alpha_{p}$ with similar interpretation for $\left(\beta_{q}\right)$. By convention the empty product is treated as unity and empty sum is treated as zero, $p, q \in \mathbb{N}_{0}$. Note: When the value of any one numerator parameter or argument or both are equal to zero then value of $\operatorname{such}_{p} F_{q}$ series will be unity.

\section{Convergence conditions of ${ }_{p} F_{q}$}

1 When $p \leq q$ then $|z|<\infty$,

2 When $p=q+1$ then $|z|<1$,

3 When $p=q+1$ and $|z|=1$ then $\Re(\omega)>0$,

4 When $p=q+1,|z|=1$ and $z \neq 1$ then $-1<\Re(\omega) \leq 0$,

where, $\omega:=\sum_{j=1}^{q} \beta_{j}-\sum_{j=1}^{p} \alpha_{j}$, and $\alpha_{j} \in \mathbb{C}(j=1,2,3, \ldots, p) ; \beta_{j} \in \mathbb{C} \backslash \mathbb{Z}_{0}^{-}(j=1,2,3, \ldots, q)$.

The Binomial expansion in terms of hypergeometric function can be written as

$$
(1-z)^{-a}={ }_{1} F_{0}\left[\begin{array}{l}
a ; z \\
-;
\end{array}\right]=\sum_{n=0}^{\infty} \frac{(a)_{n} z^{n}}{n !}
$$

where $a \in \mathbb{C}$ and $|z|<1$. 
Kampé de Fériet's double hypergeometric function ([1, p. 150, eq 29], [3, p. 112], see also [5])

$$
\mathrm{F}_{\ell: m ; n}^{p: q ; k}\left[\begin{array}{l}
\left(a_{p}\right):\left(b_{q}\right) ;\left(c_{k}\right) ; \\
\left(\alpha_{\ell}\right):\left(\beta_{m}\right) ;\left(\gamma_{n}\right) ;
\end{array} x, y\right]=\sum_{r=0}^{\infty} \sum_{s=0}^{\infty} \frac{\prod_{j=1}^{p}\left(a_{j}\right)_{r+s} \prod_{j=1}^{q}\left(b_{j}\right)_{r} \prod_{j=1}^{k}\left(c_{j}\right)_{s}}{\prod_{j=1}^{\ell}\left(\alpha_{j}\right)_{r+s} \prod_{j=1}^{m}\left(\beta_{j}\right)_{r} \prod_{j=1}^{n}\left(\gamma_{j}\right)_{s}} \frac{x^{r}}{r !} \frac{y^{s}}{s !}
$$

where denominator parameters $\left(\alpha_{\ell}\right),\left(\beta_{m}\right),\left(\gamma_{n}\right)$ are neither zero nor negative integers.

Convergence conditions of $F_{\ell: m ; n}^{p: q ; k}$ ([10, pp. 153-157, sections $\left.3 \& 4\right]$ see also [12, p. 424,eq. 27])

1 When $p+q<\ell+m+1, p+k<\ell+n+1$ then $|x|<\infty$ and $|y|<\infty$,

2 When $p+q=\ell+m+1, p+k=\ell+n+1, p>\ell$ then $|x|^{\frac{1}{p-\ell}}+|y|^{\frac{1}{p-\ell}}<1$,

3 When $p+q=\ell+m+1, p+k=\ell+n+1, p \leq \ell$ then $\max \{|x|,|y|\}<1$.

\section{Appell's function of first kind $F_{1}$ (Special case of eq. 1.7)}

$$
F_{1}[a ; b, c ; d ; x, y]=\sum_{m, r=0}^{\infty} \frac{(a)_{m+r}(b)_{m}(c)_{r}}{(d)_{m+r}} \frac{x^{m} y^{r}}{m ! r !},
$$

where, $\max \{|x|,|y|\}<1$ and $d \in \mathbb{C} \backslash \mathbb{Z}_{0}^{-}$.

The following results will be required in our present investigation.

\section{Some reduction formulas}

The following reduction formula is available in all textbooks of integral calculus

$$
\int \cos ^{n} x d x=C+\frac{\sin x \cos ^{n-1} x}{n}+\frac{(n-1)}{n} \int \cos ^{n-2} x d x,
$$

where $C$ is the constant of the integration and $\mathrm{n}$ is positive integer.

From reduction formula (1.9) we can write

$$
\begin{aligned}
\int \cos ^{2 r+1} t d t & =C+\frac{\sin t \cos ^{2 r} t}{2 r+1}+\frac{2 r}{2 r+1} \int \cos ^{2 r-1} t d t ; \quad r \geq 0, \\
\int \cos ^{2 r} t d t & =C+\frac{\sin t \cos ^{2 r-1} t}{2 r}+\frac{(2 r-1)}{2 r} \int \cos ^{2 r-2} t d t ; \quad r \geq 1 .
\end{aligned}
$$

By the successive applications of reduction formula $(1.9)$ in the right hand side of eqs. (1.10) and (1.11) we can find integrals in finite series form containing Pochhammer symbol

$$
\begin{aligned}
\int \cos ^{2 r+1} t d t & =C+\frac{\sin t(1)_{r}}{\left(\frac{3}{2}\right)_{r}}\left(\sum_{m=0}^{r} \frac{\left(\frac{1}{2}\right)_{m} \cos ^{2 m} t}{m !}\right) ; \quad r \geq 0, \\
\int \cos ^{2 r} t d t & =C+\frac{t\left(\frac{1}{2}\right)_{r}}{r !}+\frac{\sin t \cos t\left(\frac{1}{2}\right)_{r}}{r !}\left(\sum_{m=0}^{r-1} \frac{m ! \cos ^{2 m} t}{\left(\frac{3}{2}\right)_{m}}\right) ; \quad r \geq 1 .
\end{aligned}
$$

Integrals $(1.12)$ and $(1.13)$ can be verified with the reduction formulas 1.10$)$ and (1.11) by taking $r=1,2,3,4, \ldots$ 
Double series identity [11, p. 100,eq 2.1(2)]

$$
\begin{aligned}
& \sum_{r=0}^{\infty} \sum_{m=0}^{r} \Phi(r, m)=\sum_{r=0}^{\infty} \sum_{m=0}^{\infty} \Phi(r+m, m), \\
& \sum_{r=0}^{\infty} \sum_{m=0}^{r-1} \Phi(r, m)=\sum_{r=0}^{\infty} \sum_{m=0}^{\infty} \Phi(r+m+1, m),
\end{aligned}
$$

provided that series involved are absolutely convergent.

Note: The empty sum $\sum_{m=0}^{-1} \Phi(0, m)$ is treated as zero.

Integral representation of Gauss' hypergeometric function [4, p.115, eq. 2.12(7)]

$$
{ }_{2} F_{1}\left[\begin{array}{c}
a, b ; \\
c ;
\end{array}\right]=\frac{2 \Gamma(c)}{\Gamma(b) \Gamma(c-b)} \int_{0}^{\frac{\pi}{2}} \frac{\sin ^{2 b-1} t \cos ^{2 c-2 b-1} t}{\left(1-z \sin ^{2} t\right)^{a}} d t,
$$

where, $\Re(c)>\Re(b)>0$ and $|z|<1$.

Put $c=1, b=\frac{1}{2}, a=-\frac{1}{2}$ and $z=k^{2}$ in eq. 1.16 we get complete elliptic integral of second kind [9, p.71, Q. 20]

$$
\int_{0}^{\frac{\pi}{2}} \sqrt{\left(1-k^{2} \sin ^{2} \theta\right)} d \theta=\int_{0}^{\frac{\pi}{2}} \sqrt{\left(1-k^{2} \cos ^{2} \theta\right)} d \theta=\frac{\pi}{2}{ }_{2} F_{1}\left[\begin{array}{c}
-\frac{1}{2}, \frac{1}{2} ; \\
1 ;
\end{array} k^{2}\right],
$$

where $\left|k^{2}\right|<1$.

Some hypergeometric representations(see table of Prudnikov et al.[7])

$$
\begin{aligned}
& \text { 77. p.468, entry 3] } \\
& { }_{2} F_{1}\left[\begin{array}{c}
-\frac{1}{2}, \frac{1}{2} ; \\
\frac{3}{2} ;
\end{array}\right]=\frac{1}{2}\left[\sqrt{(1-z)}+\frac{\sin ^{-1} \sqrt{z}}{\sqrt{z}}\right]
\end{aligned}
$$

[7. p.469, entry 10]

$$
{ }_{2} F_{1}\left[\begin{array}{c}
-\frac{1}{2}, 1 ; \\
\frac{3}{2} ;
\end{array}\right]=\frac{1}{2}\left[1+(1-z) \frac{\tanh ^{-1} \sqrt{z}}{\sqrt{z}}\right],
$$

[7. $p .473$, entry 76]

$$
{ }_{2} F_{1}\left[\begin{array}{c}
\frac{1}{2}, \frac{1}{2} ; z \\
\frac{3}{2} ;
\end{array}\right]=\frac{\sin ^{-1} \sqrt{z}}{\sqrt{z}},
$$

[7. $p .473$, entry 83$]$

$$
{ }_{2} F_{1}\left[\begin{array}{c}
\frac{1}{2}, 1 ; \\
\frac{3}{2} ;
\end{array} z^{2}\right]=\frac{\tanh ^{-1}(z)}{z}=\frac{1}{2 z} \ln \left(\frac{1+z}{1-z}\right), \quad|z|<1 .
$$

In this paper any values of parameters and arguments leading to the results which do not make sense are tacitly excluded. 


\section{Main formulas for curved surface area of revolution and arc-length of an ellipse}

The exact expressions of the curved surface area "C.S.A." of revolution of an arc $\widehat{A B}$ between two arbitrary points $A\left(a \cos t_{1}, b \sin t_{1}\right)$ and $B\left(a \cos t_{2}, b \sin t_{2}\right)$ lying on an ellipse $\frac{x^{2}}{a^{2}}+\frac{y^{2}}{b^{2}}=1$ about the $\mathrm{y}$-axis (minor-axis) and $\mathrm{x}$-axis(major axis) and the length of that arc $\widehat{A B}$ are obtained in terms of Gauss' hypergeometric function ${ }_{2} F_{1}$, Appell's function $F_{1}$ and Kampé de Fériet's double hypergeometric function $F_{2: 1 ; 0}^{2: 2 ; 1}$

Curved surface area of oblate spheroid obtained by revolving an arc of the ellipse about minor axis

$$
\begin{aligned}
\text { C.S.A. } & =2 a^{2} \pi\left\{\sin \left(t_{2}\right) F_{1}\left[-\frac{1}{2} ; \frac{1}{2}, 1 ; \frac{3}{2} ; e^{2} \cos ^{2} t_{2}, e^{2}\right]\right. \\
& \left.-\sin \left(t_{1}\right) F_{1}\left[-\frac{1}{2} ; \frac{1}{2}, 1 ; \frac{3}{2} ; e^{2} \cos ^{2} t_{1}, e^{2}\right]\right\} .
\end{aligned}
$$

Curved surface area of prolate spheroid obtained by revolving an arc of the ellipse about major axis

C.S.A. $=2 a b \pi\left\{\cos \left(t_{1}\right)_{2} F_{1}\left[\begin{array}{c}-\frac{1}{2}, \frac{1}{2} ; e^{2} \cos ^{2} t_{1} \\ \frac{3}{2} ;\end{array}\right]-\cos \left(t_{2}\right)_{2} F_{1}\left[\begin{array}{c}-\frac{1}{2}, \frac{1}{2} ; e^{2} \cos ^{2} t_{2} \\ \frac{3}{2} ;\end{array}\right]\right\}$.

Arc-length of an arc $\mathrm{AB}$ of the ellipse

$$
\begin{aligned}
\widehat{A B} & =a t_{2}{ }_{2} F_{1}\left[\begin{array}{c}
-\frac{1}{2}, \frac{1}{2} ; \\
1 ;
\end{array} e^{2}\right]-\frac{a e^{2} \sin t_{2} \cos t_{2}}{4} \mathrm{~F}_{2: 1 ; 0}^{2: 2 ; 1}\left[\begin{array}{c}
\frac{1}{2}, \frac{3}{2}: 1,1 ; 1 ; \\
2,2: \frac{3}{2} ;-;
\end{array} e^{2} \cos ^{2} t_{2}, e^{2}\right]- \\
& -a t_{12} F_{1}\left[\begin{array}{c}
-\frac{1}{2}, \frac{1}{2} ; \\
1 ;
\end{array} e^{2}\right]+\frac{a e^{2} \sin t_{1} \cos t_{1}}{4} \mathrm{~F}_{2: 1 ; 0}^{2: 2 ; 1}\left[\begin{array}{c}
\frac{1}{2}, \frac{3}{2}: 1,1 ; 1 ; \\
2,2: \frac{3}{2} ;-;
\end{array} e^{2} \cos ^{2} t_{1}, e^{2}\right],(2.3)
\end{aligned}
$$

where $a$ is the length of semi-major axis and $e$ is the eccentricity of the ellipse. Here, all three series $F_{1},{ }_{2} F_{1}$ and $F_{2: 1 ; 0}^{2: 2 ; 1}$ are convergent since $e<1$ and $e \cos t<1$. Therefore, our expressions given in eqs. (2.1), 2.2) and (2.3) are convergent and are believed to be new.

Derivation of formula 2.1

Consider the equation of ellipse

$$
\frac{x^{2}}{a^{2}}+\frac{y^{2}}{b^{2}}=1 \text { (Cartesian form) }
$$

when, $a>b$, then $b^{2}=a^{2}\left(1-e^{2}\right)$ or $\frac{a^{2}-b^{2}}{a^{2}}=e^{2}<1, a$ and $b$ are semi-major and semiminor axes of the ellipse respectively and $e(<1)$ is called the eccentricity of ellipse.

Its parametric form is given by

$$
x=a \cos t, \quad y=b \sin t .
$$

Since given ellipse 2.4] is symmetrical about $x$ - axis and $y$ - axis both, without any loss of generality, we shall find the arc-length between two arbitrary points lying in positive quadrant only(i.e. $0 \leq t_{1}<t_{2} \leq \frac{\pi}{2}$ ). 


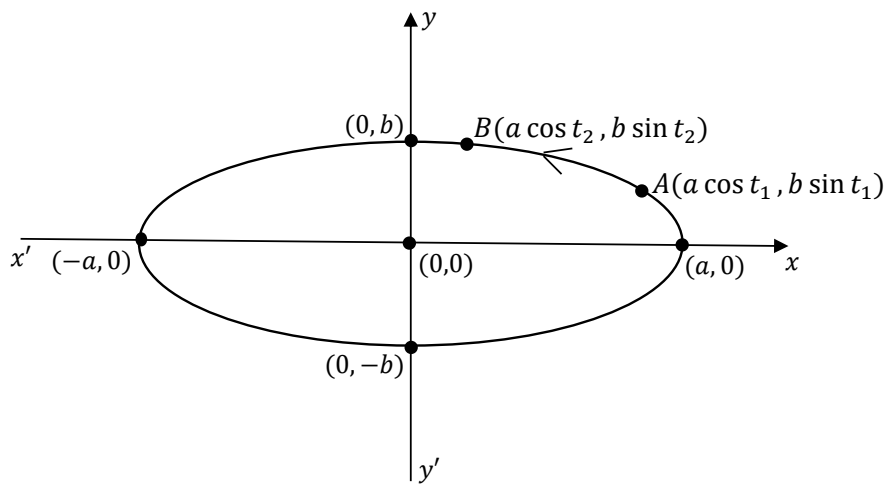

Fig. 1 Ellipse $\frac{x^{2}}{a^{2}}+\frac{y^{2}}{b^{2}}=1$

The curved surface area of revolution of an arc about the $y$ - axis between two arbitrary points $A$ and $B$ lying on any parametric curve is given by

$$
S=2 \pi \int_{t_{1}}^{t_{2}} x \sqrt{\left\{\left(\frac{d x}{d t}\right)^{2}+\left(\frac{d y}{d t}\right)^{2}\right\}} d t .
$$

Put $\frac{d x}{d t}=-a \sin t, \frac{d y}{d t}=b \cos t$ in eq. (2.6) and integrating w. r. to $t$ over the interval $\left[t_{1}, t_{2}\right]$ s.t. $0 \leq t_{1}<t_{2} \leq \frac{\pi}{2}$.

The curved surface area of Oblate spheroid generated by the revolution of an arc about the $y$-axis (i.e. minor axis) between two arbitrary points $A$ and $B$ lying on ellipse is given by

$$
\begin{aligned}
\text { C.S.A. } & =2 a \pi \int_{t_{1}}^{t_{2}} \cos t \sqrt{\left(a^{2} \sin ^{2} t+b^{2} \cos ^{2} t\right)} d t=2 a \pi \int_{t_{1}}^{t_{2}} \cos t \sqrt{\left\{a^{2}-\left(a^{2}-b^{2}\right) \cos ^{2} t\right\}} d t, \\
& =2 a^{2} \pi \int_{t_{1}}^{t_{2}} \cos t \sqrt{\left\{1-\left(\frac{a^{2}-b^{2}}{a^{2}}\right) \cos ^{2} t\right\}} d t=2 a^{2} \pi \int_{t_{1}}^{t_{2}} \cos t \sqrt{\left(1-e^{2} \cos ^{2} t\right)} d t, \\
& =2 a^{2} \pi \int_{t_{1}}^{t_{2}}(\cos t){ }_{1} F_{0}\left[\begin{array}{l}
-\frac{1}{2} ; \\
-;
\end{array} e^{2} \cos ^{2} t\right] d t ; \quad \text { since } \quad e^{2} \cos ^{2} t<1, \quad \forall \quad t, \\
& =2 a^{2} \pi \sum_{r=0}^{\infty} \frac{e^{2 r}\left(-\frac{1}{2}\right)_{r}}{r !} \int_{t_{1}}^{t_{2}} \cos ^{2 r+1} t d t .
\end{aligned}
$$

Using integral (1.12) in eq. (2.7) we get,

$$
\begin{aligned}
S & =2 a^{2} \pi \sum_{r=0}^{\infty} \frac{e^{2 r}\left(-\frac{1}{2}\right)_{r}}{r !}\left\{\frac{\sin t(1)_{r}}{\left(\frac{3}{2}\right)_{r}} \sum_{m=0}^{r} \frac{\left(\frac{1}{2}\right)_{m} \cos ^{2 m} t}{m !}\right\}_{t_{1}}^{t_{2}} \\
& =2 a^{2} \pi\left\{(\sin t) \sum_{r=0}^{\infty} \sum_{m=0}^{r} \frac{e^{2 r}\left(-\frac{1}{2}\right)_{r}}{\left(\frac{3}{2}\right)_{r}} \frac{\left(\frac{1}{2}\right)_{m} \cos ^{2 m} t}{m !}\right\}_{t_{1}}^{t_{2}}
\end{aligned}
$$


Now using double series identity (1.14) in eq. (2.8), we get

$$
S=2 a^{2} \pi\left\{(\sin t) \sum_{r=0}^{\infty} \sum_{m=0}^{\infty} \frac{e^{2(r+m)} \cos ^{2 m} t\left(-\frac{1}{2}\right)_{r+m}\left(\frac{1}{2}\right)_{m}}{m !\left(\frac{3}{2}\right)_{m+r}}\right\}_{t_{1}}^{t_{2}}
$$

or

$$
\text { C.S.A. }=2 a^{2} \pi\left\{(\sin t) \mathrm{F}_{1}\left[-\frac{1}{2} ; \frac{1}{2}, 1 ; \frac{3}{2} ; e^{2} \cos ^{2} t, e^{2}\right]\right\}_{t_{1}}^{t_{2}} .
$$

After simplification we get our required result (2.1). Simillarly, on using eqs. (1.13) and (1.15) we have easily derived the expressions given in eqs. (2.2) and (2.3).

\section{Case Study}

Case 1. On putting $t_{1}=0$ and $t_{2}=\frac{\pi}{2}$ in eq. 2.1) we get curved surface area of hemi-oblate spheroid in the form:

$$
\begin{aligned}
\text { C.S.A. } & =2 a^{2} \pi \mathrm{F}_{1}\left[-\frac{1}{2} ; \frac{1}{2}, 1 ; \frac{3}{2} ; 0, e^{2}\right], \\
& =2 a^{2} \pi_{2} F_{1}\left[\begin{array}{c}
-\frac{1}{2}, 1 ; \\
\frac{3}{2} ;
\end{array} e^{2}\right], \\
& =a^{2} \pi\left\{1+\left(1-e^{2}\right) \frac{\tanh ^{-1}(e)}{e}\right\}, \\
& =a^{2} \pi\left\{1+\left(\frac{1-e^{2}}{2 e}\right) \ln \left(\frac{1+e}{1-e}\right)\right\} .
\end{aligned}
$$

Therefore, total curved surface area of oblate spheroid is given by

$$
2 a^{2} \pi\left\{1+\left(\frac{1-e^{2}}{2 e}\right) \ln \left(\frac{1+e}{1-e}\right)\right\} .
$$

When $e \rightarrow 0$ ellipse tends to circle, therefore total curved surface area of resulting sphere is $4 \pi a^{2}$, where $a$ is the radius of the sphere (on applying L' Hôpital's rule for $\frac{0}{0}$ form in eq. (3.2)).

Case 2. On putting $t_{1}=0$ and $t_{2}=\frac{\pi}{2}$ in eq. 2.2 we get curved surface area of hemiprolate spheroid in the form:

$$
\text { C.S.A. }=2 a b \pi_{2} F_{1}\left[\begin{array}{c}
-\frac{1}{2}, \frac{1}{2} ; \\
\frac{3}{2} ;
\end{array} e^{2}\right]=a b \pi\left\{\sqrt{\left(1-e^{2}\right)}+\frac{\sin ^{-1}(e)}{e}\right\} .
$$

Therefore, total curved surface area of prolate spheroid is given by

$$
2 a b \pi\left\{\sqrt{\left(1-e^{2}\right)}+\frac{\sin ^{-1}(e)}{e}\right\} .
$$

When $e \rightarrow 0$ ( or $b \rightarrow a$ ) ellipse tends to circle, therefore total curved surface area of resulting sphere is $4 \pi a^{2}$, where $a$ is the radius of the sphere (on applying L' Hôpital's rule for $\frac{0}{0}$ form in eq. (3.4)). 
Case 3. To obtain the perimeter of the ellipse we shall put $t_{1}=0$ and $t_{2}=\frac{\pi}{2}$ in eq. 2.3 and multiply by 4 , we get the perimeter $(p)$

$$
p=2 a \pi_{2} F_{1}\left[\begin{array}{c}
-\frac{1}{2}, \frac{1}{2} ; \\
1 ;
\end{array} e^{2}\right],
$$

where $a$ is the semi-major axis and $e$ is the eccentricity of the ellipse.

According to Kepler's first law: "Each planet's path around the Sun is an ellipse with the Sun at one focus of the ellipse [6, p. 56]". So the results of this paper can also be used in astronomy.

The length of semi-major axis $(a)$ and orbital eccentricity $(e)$ of each planet is taken from NASA website then perimeter $(p)$ of each orbit is calculated by our result (3.5) which is shown in last column of table 1

\begin{tabular}{|c|c|c|c|c|}
\hline S. No. & Name of Planet & $\begin{array}{c}\text { Length of Semi-Major Axis }(a) \\
\text { (in K.M.) }\end{array}$ & Orbital Eccentricity $(e)$ & $\begin{array}{c}\text { Perimeter of Orbit }(p) \\
\text { (in K.M.) }\end{array}$ \\
\hline 1 & Mercury & $5.791 \times 10^{7}$ & 0.2056 & $3.59983 \times 10^{8}$ \\
\hline 2 & Venus & $1.0821 \times 10^{8}$ & 0.0068 & $6.798956 \times 10^{8}$ \\
\hline 3 & Earth & $1.496 \times 10^{8}$ & 0.0167 & $9.39899 \times 10^{8}$ \\
\hline 4 & Mars & $2.2792 \times 10^{8}$ & 0.0934 & $1.428935 \times 10^{9}$ \\
\hline 5 & Jupiter & $7.7857 \times 10^{8}$ & 0.0484 & $4.889033 \times 10^{9}$ \\
\hline 6 & Saturn & $1.43353 \times 10^{9}$ & 0.0542 & $9.000516 \times 10^{9}$ \\
\hline 7 & Uranus & $2.87246 \times 10^{9}$ & 0.0472 & $1.803814 \times 10^{10}$ \\
\hline 8 & Neptune & $4.49506 \times 10^{9}$ & 0.0086 & $2.824277 \times 10^{10}$ \\
\hline
\end{tabular}

Table 1 Table for perimeter of each orbit

If $b=a$, then eccentricity $e$ will be zero and the equation of ellipse $\frac{x^{2}}{a^{2}}+\frac{y^{2}}{b^{2}}=1$ reduces to the equation of circle $x^{2}+y^{2}=a^{2}$ therefore, the perimeter $p$ given by eq. 3.5 becomes $p=2 a \pi$ which is the circumference of the circle, since ${ }_{2} F_{1}\left[\begin{array}{c}-\frac{1}{2}, \frac{1}{2} ; 0 \\ 1 ;\end{array}\right]=1$.

\section{Remark}

We have also derived some formulas [8] for arc-length between two arbitrary points lying on hyperbola $\frac{x^{2}}{a^{2}}-\frac{y^{2}}{b^{2}}=1$ and rectangular hyperbola $x y=c^{2}$.

\section{Conclusion}

We conclude our present investigation by observing that solutions of such problems can be obtained in an analogous manner. 


\section{References}

1. Appell, P. and Kampé de Fériet, J.: Fonctions hypergéométriques et hypersphériques: Polynômes d'Hermite, Gauthier-Villars, Paris, (1926).

2. Bailey, W.N.: Generalised Hypergeometric Series, Cambridge Math. Tract No. 32, Cambridge University Press, Cambridge 1935: Reprinted by Stechert-Hafner, New York, (1964).

3. Burchnall, J.L. and Chaundy, T.W.: Expansions of Appell's double hypergeometric functions (II), Quart. J. Math. Oxford Ser., 12, 112-128 (1941).

4. Erdélyi, A. Magnus, W., Oberhhettinger, F. and Tricomi, F. G.: Higher Transcendental Functions, Vol. I, McGraw-Hill Book Company, New York/Toronto/ London, (1953).

5. Kampé de Fériet J.: Les Fonctions hypergéométriques d'ordre supérieur à deux variables, C.R. Acad. Sci. Paris, 173, 401-404 (1921).

6. Kuhn, K.F. and Koupelis, T.: In Quest of the universe, Fourth Edition, Jones and Bartlett Publishers. Boston/Toronto/London/Singapore, (2004).

7. Prudnikov, A.P., Brychkov, Yu. A. and Marichev, O.I.: Integrals and series Vol. 3: More Special Functions. Nauka, Moskow, 1986. Translated from the Russian by G.G. Gould, Gordan and Breach Science Publishers, New York, Philadelphia, London, Paris, Montreux, Tokyo, Melbourne, (1990).

8. Qureshi, M. I., Akhtar, N. and Ahamad, D.: Analytical expressions for arc-length of hyperbola: A hypergeometric approach, IJCER, 9(6), 44-52 (2019).

9. Rainville, E.D.: Special Functions, The Macmillan Company, New York 1960, Reprinted by Chelsea Publishing Company, Bronx, New York, (1971).

10. Srivastava, H.M. and Daoust, M. C.: A note on the convergence of Kampé de Fériet's double hypergeometric series, Math. Nachr., 53, 151-159 (1972).

11. Srivastava, H.M. and Manocha, H.L.: A Treatise on Generating Functions, Halsted Press (Ellis Harwood Limited, Chichester, U.K.), John Wiley \& Sons, New York, Chichester, Brisbane and Toronto, (1984).

12. Srivastava, H.M. and Panda, R.: An integral representation for the product of two Jacobi polynomials, J. London Math. Soc. 12 (2), 419-425 (1976). 